\title{
Exploring the Benefits of Digital Storytelling for Enhancing Teaching and Learning
}

\author{
Hary Soedarto Harjono ${ }^{1, *}$ Mujiyono Wiryotinoyo ${ }^{1}$ \\ ${ }^{1}$ Universitas Jambi \\ *Corresponding author. Email: hary.soedarto@unja.ac.id
}

\begin{abstract}
This paper presents excerpts from a literature study, which is part of a larger study to explore the benefits of digital storytelling (DST) in strengthening student comprehension, students' engagement and motivation in higher education. Specifically, the study is focused on the use of DST as a learning activity and a tool for evaluating student learning outcomes in undergraduate lectures. In this context, DST as an activity focuses on students' action in creating DST in a classroom-based project situation, both individually and collaboratively. As an evaluation tool, DST is used as a media of learning reflection in certain topics. From the results of the study, it can be found that the use of DST as a classroombased project activity may enhance the teaching and learning process to be more interesting, motivates students to engage more in learning and comprehend more deeply the learning material. In addition, reflection in the form of DST is preferable compared to paper-based written reflection.
\end{abstract}

Keywords: digital storytelling, teaching and learning, students' engagement.

\section{INTRODUCTION}

The tradition of oral storytelling has existed since the beginning of human civilization. Recently, the oral tradition has evolved and run to a transformation, both in the medium of delivery of the storytelling and its function in human civilization. One form of transformation is digital storytelling, which essentially tells a personal story or narrative using digital technology to construct meaning [1]. In this sense, DST is a short video which is a combination of multimedia consisting of images, videos, accompanying music, and narration that can be used to tell and reflect on unique experiences of human life. In the context of education, DST has been widely used by educators as well as learners to improve the quality of the learning process and outcomes.

DST has developed into a "new pedagogical tool" which is supported by the rapid development of digital technology which includes software in image processing, video, text, and multimedia in general. DST facilitates the convergence of four student-centered learning strategies (Barret, 2005), specifically in strengthening learner involvement in learning, allows learners to reflect on understanding topics that are studied in a deep-learning situation. In addition, DST can be implemented as a student's tasks situated in a project-based learning environment. Moreover, to create and develop DST requires students' skills in technology integration.

As a new pedagogical tool, many studies that have been carried out by educational researchers found that DST has many positive impacts that can be applied and implemented for enhancing and deepening learning at all levels of education and all fields of studies. Empirically, the use of DST in learning process does not only help learners in mastering learning material [2] [3] and technology skills [4], but also increases learning motivation [5][6][7] and practicing creative thinking skills [8].

From the studies above, it can be summarized that DST provides many benefits in an educational context. 
Therefore, this paper will explore the benefits of DST in strengthening learning processes and outcomes in a more extensive overview on research findings in teaching and learning context. The overview presented here is part of the author's research which focuses on the impact of DST on enhancing student comprehension and motivation in learning in one public university in Indonesia.

\section{DST AND DEEP-LEARNING}

A strong trend in research findings on the use of DST in learning leads to strengthening learning process and learning outcomes. For instance, Ryan \& Aasetre [6] conducted research on the integrative use of DST in Geography teaching and learning activities in the context of higher education. An important finding from this study is that DST may enhance deep-learning, which indicates understanding and creative use of knowledge in new contexts. In addition, involving students in a project to create DST situated in classroom activities also motivates students to learn more deeply. In this context, DST allows students to internalize the abstract concepts they learn to be more concrete because of the help of technology integration by combining images, text, and sound in the process of DST creation.

As it implied from Ryan \& Aasetre [6], by using DST as learning media, the learning process becomes more interesting and more realistic with the support of technology integration in the form of DST which is used for learning. Emotional involvement is stronger in the learning process because abstract concepts can be visualised, making it easier to understand. Deeper comprehension of learning content is possible because emotional involvement is very important in strengthening deep learning.

Furthermore, Sadik, [5] found that as a technologyintegrated approach, DST can improve learning. In this study, which involved teachers and students in Egypt, students were assigned to create DST using MS Photo Story software. After the students finished the digital stories project, they presented them in class. Furthermore, the research data were obtained from quantitative and qualitative instruments in the form of a digital story evaluation rubric, observation instruments to observe technology integration, and interviews to evaluate the effectiveness of DST in implementing learning. In the context of this study, it was further explored the extent to which students were involved in authentic learning tasks with DST; how effective the DST approach is in facilitating teachers to integrate technology in learning; and the focus and views of teachers with regard to the implementation and integration of DST in learning.
Sadik's [5] findings showed that overall students can complete DST assignments well, meaning that the DST fulfil pedagogical and technological attributes. In addition, from the results of classroom observations and interviews, it can be shown that although problems were found in the process and implementation of the DST, teachers believe that activities in the classroom by integrating technology in learning through DST may improve students' comprehension of learning material contained in the curriculum. Furthermore, based on interviews data, it was revealed that there was a desire of the teachers to transform the pedagogy and curriculum by integrating DST in the classroom learning.

Likewise, Yang, Chen \& Hung's study [8] shows that DST is effective in improving creative thinking skills and English-speaking skills in the context of English as a foreign language. In the context of this research, DST is an interdisciplinary project integrating English language lectures with computers. English lecture activities lead to designing content, while computer lectures are aimed at designing DST. At the computer lecture, students are assigned to design multimedia in the form of digital stories. Participants create DST collaboratively for 8 weeks in small groups in the target language, English. Furthermore, two quasi-experimental groups with a pretest-posttest design were compared their learning outcomes. The results showed that DST was effective in facilitating learners in improving their creative thinking skills and English-speaking proficiency. Shortly, DST may facilitate learners to enhance learning processes and outcomes.

\section{DST, STUDENT'S ENGAGEMENT AND TECHNOLOGICAL SKILLS}

In various contexts, studies on DST show a strong tendency towards positive findings in strengthening learning process, outcomes and learner motivation in engaging with learning activities. The Global Sharing Pedagogy (GSP) approach through qualitative research found that DST strongly supports the development of the 21st-century skills, particularly when DST is situated in a collaborative learning context. The results of this study reveal that the use of DST is very interesting to students, motivates and engages students emotionally in learning so that they are challenged to carry out more intensive learning activities.

Similar findings can be found from Hava's [7] study. In the experimental research, Hava [7] explores the effects of DST on motivation and satisfaction levels in the education of English as a foreign language. In addition, students' opinions about the use of DST in the learning environment were also examined. In this study, 
the students were assigned to create DST, which in the process took 9 weeks. Before and after the process of DST creation, students were measured their level of motivation and satisfaction in learning. The results showed that there was a significant improvement in selfconfidence and personal use of DST after the DST creation. In addition, from the research it can be shown that DST is useful for facilitating vocabulary learning, writing and speaking skills in the context of learning English as a foreign language. Overall, it can be shown that DST can be used as an important tool not only in language learning, but also to support students' language skills and skills in digital literacy.

In a different context of study, research conducted by Coggin et al [9] presents an example of using DST critically to imagine future classrooms. The qualitative research design was used by positioning the DST as a performance and analysing the art form to consider how prospective teachers as participants in this study can use the text to imagine and concretize it. The critical case in this study shows how prospective teachers interpret their personal experiences, their image of the ideal teacher, assignments related to teaching methods, and personal struggles that are sometimes not in line with teaching and learning activities. The findings show that DST expands the reflective practice of prospective teachers in an environment that supports teacher education programs.

Student experiences based on reflections on pedagogical transition as outlined in the form of DST at a higher education institution in the UK. The qualitative data were obtained from the analysis of the DST created by participating students. The results of this study indicate that DST is more effectively used for reflection compared to reflection in paper-based writing. In addition, DST provides rich qualitative data for evaluation and monitoring as well as for strengthening pedagogical and curriculum outcomes.

Tanrikulu [3]'s research aims to identify students' perceptions of how collaborative use of DST, which is used in writing classes, affects their writing skills. By using a qualitative research design, this study applies an action research model. The study was conducted in two different classes with first-year students studying at the Turkish Ministry of Education (N 1/4 61). Students have produced two different DSTs during the lecture. The first DST is created individually, and the second one collaboratively. Students participate actively in producing and providing criticism and suggestions at the stages of the DST creation process. The researchers themselves, student diaries and focus group interviews were used as data collection tools. The findings show that
DST improves students' writing skills. This is reflected in student perceptions that show that DST in the form of multimedia has a positive effect on writing skills and mastery of technology skills.

In line with the results of Tanrikulu [3] and Nguyen, Hwang, Pham \& Pham [2], research findings in the EFL context also show that the use of DST in an authentic context motivates learners to share experiences that not only improve writing skills in English, but also increase self-confident to use English in real situations. To sum up, the use of DST in learning may enhance students' engagement, motivation and technological skills.

\section{CONCLUSION}

The findings on DST showed many positive benefits of DST for teaching and learning purposes. DST can be used as a medium, material, and tool for evaluating learning in various fields of study and various levels of education.

As a learning medium, DST is effective for visualizing abstract learning topics to be more interesting, concrete, and easier for understanding. As material, DST can be used as learning material that involves students actively in deep-learning. Students can be involved deeply in the process of creating DST, either individually or collaboratively with guidance from the teacher to develop learning materials that fit with the curriculum. As an evaluation tool, DST is proven to be more challenging and effective to measure understanding of learning material through reflection expressed in the form of DST.

To summarize, DST may facilitate learners for deeplearning reflection on whatever they did and learned. Particularly, applying DST in learning can enhance learning processes and outcomes, strengthen learning engagement and motivation, increase digital literacy, and develop technology skills.

\section{ACKNOWLEDGMENT}

Thanks go to the PNBP Research Funding Project of Universitas Jambi, Indonesia that has funded this study.

\section{REFERENCES}

[1] J Lambert. (2013). Digital Storytelling: Capturing lives, creating community. New York: Routledge.

[2] T H Nguyen. W Y Hwang, X L Pham \& T Pham (2020):Self-experienced storytelling in an authentic context to facilitate EFL writing, 
Computer AssistedLanguage Learning, DOI: 10.1080/09588221.2020.1744665.

[3] F Tanrikulu. (2020): Students' perceptions about the effects of collaborative digital storytelling on writing skills, Computer Assisted Language Learning,

DOI: 10.1080/09588221.2020.1774611.

[4] N Churchill, (2020). Development of students' digital literacy skills through digital storytelling with mobile devices, Educational Media International,

DOI: 10.1080/09523987.2020.1833680.

[5] A Sadik. (2008). Digital Storytelling: a meaningful technology-integrated approach for engaged student learning, Education Tech Research Development, 56: 487-506, DOI: 10.1007/s11423-008-9091-8.

[6] A W Ryan. \& J Aasetre. (2020): Digital storytelling, student engagement and deep learning in Geography, Journal of Geography in Higher Education, DOI: $\underline{10.1080 / 03098265.2020 .1833319}$.

[7] K Hava. (2019): Exploring the role of digital storytelling in student motivation and satisfaction in EFL education, Computer Assisted Language Learning, DOI: 10.1080/09588221.2019.1650071

[8] Y T C Yang, Y C Chen \& H T Hung (2020): Digital storytelling as an interdisciplinary project to improve students' English speaking and creative thinking, Computer Assisted Language Learning,

DOI: 10.1080/09588221.2020.1750431.

[9] L S Coggin. S Daley., J Sydnor. \& T R Davis. (2019): Imagining my ideal: a critical case study of digital storytelling as reflective practice, Reflective Practice, DOI: 10.1080/14623943.2018.1538949. 